\title{
Effect of tactual scanning mode on braille and shape recognition
}

\author{
MORTON A. HELLER, DANETTE K. SCROFANO, and KIMBERLY D. NESBITT \\ Winston-Salem State University, Winston-Salem, North Carolina
}

\begin{abstract}
Two experiments studied the influence of tactual scanning mode and finger locus on pattern recognition. In Experiment 1, observers made visual matches to braille characters that were examined with either lateral scanning or free tracing. In Experiment 2, the same tactile scanning modes were used to explore embossed shapes. Performance was comparable with the extreme fingertip and the fingerpad. However, lateral scanning was inferior to tracing and yielded much lower recognition scores in both experiments.
\end{abstract}

The purpose of the present study was to examine the effect of tactual scanning mode on braille and shape recognition. It is very difficult for many late-blind persons to learn to read braille with any proficiency. Large numbers of individuals never learn to read braille, and others read very slowly (Foulke, 1982). Prior research by the first author (Heller \& Mitchell, 1985) showed superior braille recognition when the extreme fingertip was used to examine stimuli. However, that experiment did not properly control for scanning mode, and other research has shown superior haptic performance with free exploration (Heller, 1986), tactile scanning (Phillips, Johnson, \& Browne, 1983), or minimal movement of the fingerpad (Loomis, 1985). Lateral scanning is normally employed for braille reading, and tracing movements are typically discouraged (Foulke, 1982).

In Experiment 1, observers in the lateral scan conditions were restricted to lateral, side-to-side movements when touching braille. Other subjects were permitted to trace the stimuli. Some individuals were allowed the use of the extreme fingertip, and others were restricted to the fingerpad (the tip of the finger was covered with tape, see Heller \& Mitchell, 1985). Experiment 2 used larger, embossed shapes, but omitted the fingertip, lateral-scan condition. We expected that free tracing would prove superior, and that the extreme fingertip would yield better performance than the fingerpad.

\section{EXPERIMENT 1}

Subjects in Experiment 1 made visual matches to tangible braille. Independent groups of subjects used the fingertip or the fingerpad, and lateral scanning or free tracing.

Preparation of this report and some of the research were supported by NIH MBRS Grant 2 SO6 RR-08040. Faith Heller provided helpful comments on an earlier version of this manuscript. I wish to thank Emerson Foulke for the loan of a special slate that was used to emboss the forms used in Experiment 2. Address correspondence to Morton A. Heller, Department of Psychology, Winston-Salem State University, Winston-Salem, NC 27110.

\section{Method}

Subjects. There were 56 subjects in Experiment 1, with 14 in each of four groups. Equal numbers of males and females were randomly assigned to each condition. All subjects were experimentally naive.

Stimuli. The tangible stimuli were embossed braille patterns representing the letters A-J (standard braille uses a $3 \times 2$ matrix of dots and is about $6 \mathrm{~mm}$ high). The braille characters A-J are less than $4 \mathrm{~mm}$ high. A standard Perkins brailler was used to emboss the braille on manila braille paper. The 10 standard braille characters were presented five times each in a random arrangement, for $\mathbf{5 0}$ items in all. The stimuli were arranged in five rows with nine characters per row, and a final row that contained only five patterns. A drawing of the braille code and letter equivalents was present throughout. A Masonite screen with a clothcovered opening at the base was used to prohibit sight of the tactually examined braille.

Design and Procedure. The experiment was a two-way independent groups analysis of variance. The factors were finger locus (tip or fingerpad) and scanning mode (tracing or lateral scan). Independent groups of subjects were limited to the use of the extreme tip of the finger or the fingerpad (see Figure 1). In lateral tracing, a straight plastic guide was placed above each line of braille. The subjects placed their fingertips against this guide as they moved the preferred index finger back and forth, from side-to-side, over each braille character. The subjects were timed from the beginning to the end of each line of braille. Time limits were not imposed, and feedback was not given. The subjects were not allowed to move their fingers up and down in the lateral scan condition. In the tracing mode, the subjects were told they were free to trace the patterns with the fingertip or the fingerpad, as appropriate The extreme tip of the finger was covered with tape in the fingerpad conditions.

\section{Results and Discussion}

Braille recognition was superior with tracing procedures $[F(1,52)=18.5, p<.001]$, but finger locus had no effect on perception $(F<1$; see Table 1$)$. A second analysis of variance on time showed no effect of finger locus $(F<1)$ and a nonsignificant effect of scan mode on response latency $[F(1,52)=1.6, p>.05]$. The interaction was also nonsignificant $(F=1.03)$. These results indicate that the fingerpad is probably a better skin surface for normal braille reading; the use of the extreme tip of the finger may exaggerate the tendency of subjects to get lost when reading braille text (see Heller, 1985). Free tracing is clearly superior to lateral scanning, and may be necessary for the novice braille reader. Tracing slows down reading, however, and should certainly be discouraged in the more advanced braille reader. 

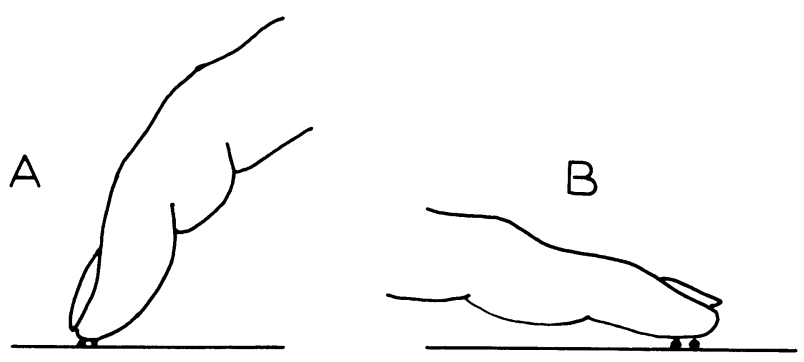

Figure 1. This figure shows the fingertip locus (A) and the fingerpad locus (B).

\section{EXPERIMENT 2}

Experiment 2 used embossed stimuli somewhat larger than and different from those in Experiment 1. It was expected that tracing would produce better pattern recognition than lateral scanning.

\section{Method}

Subjects. There were 36 subjects in Experiment 2. None had served in Experiment 1.

Stimuli and Apparatus. The stimuli were simple shapes comprising braille-like dots embossed on plastic cards. The shapes were produced with a $6 \times 6$ matrix of dots. The largest shape was about $12.5 \times 12.5 \mathrm{~mm}$. Spacing between the dots that made up each shape was similar to that between standard braille dots. The eight shapes (cross, $\mathrm{X}$, right triangle, square, rectangle, hexagon, circle, and ellipse) were presented three times each in a random arrangement. The shapes were arrayed in four horizontal lines with six shapes in each line. There were horizontal spaces of about $12.5 \mathrm{~mm}$ between the shapes and approximately the same vertical spaces between the lines. As in Experiment 1, a Masonite baffle was used to prohibit sight of the embossed shapes during tactual exploration. A visual matching array was available during haptic exploration. The array had numbered drawings of the tangible stimuli and allowed subjects to name the picture that corresponded to the shape that was touched.

Design and Procedure. There were three independent groups in this experiment. Two groups of subjects used the fingerpad for either lateral scanning or tracing of the shapes. A third group used the fingertip for tracing. (It was not possible for most subjects to examine the stimuli using a single lateral scan with the fingertip, owing to the small size of that skin surface and the large size of the patterns.) The procedure was similar to that of Experiment 1. A visual matching array was available as the subjects touched the shapes. The matching array consisted of line drawings of the patterns and numbers; the subjects named the number corresponding to the shape that was touched. Feedback was not given, and time limits were not imposed. As in Experiment 1, the subjects were timed on each line of shapes.

\section{Results and Discussion}

Shape recognition was degraded when subjects were limited to lateral scanning $[F(2,33)=5.7, p<.01]$.

Table 1

Mean Number Correct, Standard Deviation, and Mean Time Per Braille Character (in sec) in Experiment 1

\begin{tabular}{cccccccc}
\hline & \multicolumn{5}{c}{ Mode } \\
\cline { 2 - 5 } \cline { 5 - 7 } Locus & $M$ & $S D$ & Time & & $M$ & $S D$ & Time \\
\hline Fingertip & 38.6 & 7.9 & 9.0 & & 28.5 & 9.2 & 9.2 \\
Fingerpad & 38.0 & 9.0 & 7.7 & & 26.5 & 11.3 & 9.5 \\
\hline
\end{tabular}

Note-The maximum score possible $=50$.
Mean number correct (out of a possible score of 24) for tracing with the fingertip, tracing with the fingerpad, and lateral scanning with the fingerpad was 20.67 ( $S D=$ $2.35), 19.5(S D=3.15)$, and $16.1(S D=4.5)$, respectively. A second analysis of variance on time showed a nonsignificant effect of mode $(F<1)$.

\section{GENERAL DISCUSSION}

Two-dimensional tactile pattern recognition is more difficult when subjects are limited to lateral scanning. The results of the present experiments show that the fingerpad is as efficient as the extreme fingertip, given stimuli such as braille or small, two-dimensional shapes. These results are consistent with Heller's (1986) prior report of the superiority of active tracing for braille recognition. In that study, passive lateral scanning and passive vertical scanning yielded identical, but low performance.

Tracing may aid tactile pattern recognition by allowing sequential sensory input. The naive, sighted subject may have difficulty coping with much tactile spatial information in short periods of time. Furthermore, sequential input may facilitate the construction of an outline image (Heller, 1985). This is difficult for braille and the sense of touch (Heller, 1987), but may be demanded (and induced) by the visual matching task. Tactile tracing allows the observer to translate two-dimensional cutaneous input into kinesthetic movement patterns. For example, many observers will engage in L-shaped tracing movements when scanning the braille character for the letter $\mathrm{H}($ (.). Similar tracing occurs with other configurations, as the cutaneous input "drives" tactile scanning. Thus, observers attempt to follow the outlines of braille patterns with their fingertips. Nolan and Kederis (1969) assumed that individuals construct an outline impression of braille. Heller (1985) showed that naive, sighted persons engage in these scanning behaviors: individuals were able to make visual matches to braille characters (they could not see or touch) when limited to visual observation of other naive persons touching braille.

It is not possible, of course, to discount the possibility that the tracing procedure was superior to lateral scanning because observers could simply have used vertical scans (Thurlow, 1986). Thurlow found lateral scanning was inferior to vertical scanning; however, he used a passive procedure in which the subject's finger was moved and the patterns were much larger than normal braille (about $13 \mathrm{~mm}$ high). It seems unlikely, though, that an advantage for vertical scanning could explain the present results with standard braille (see Heller, 1986).

\section{REFERENCES}

FoulKe, E. (1982). Reading braille. In W. Schiff \& E. Foulke (Eds.), Tactual perception: A sourcebook. New York: Cambridge University Press.

Heller, M. A. (1985). Tactual perception of embossed Morse code and braille: The alliance of vision and touch. Perception, 14, 563-570.

HeLler, M. A. (1986). Active and passive tactual braille recognition. Bulletin of the Psychonomic Society, 24, 201-202.

Heller, M. A. (1987). The effect of orientation on visual and tactual braille recognition. Perception, 16, 291-298.

Heller, M. A., \& MrTCHELL, B. Y. (1985). Helping new braille readers: Effects of spacing, finger locus, and gloves. Perceptual and Motor Skills, 61, 363-369.

LoomIs, J. M. (1985). Tactile recognition of raised characters: A parametric study. Bulletin of the Psychonomic Society, 23, 18-20.

Nolan, C. Y., \& Kederis, C. J. (1969). Perceptual factors in Braille word recognition. New York: American Printing House for the Blind.

Phillips, J. R., Johnson, K. O., \& Browne, H. M. (1983). A comparison of visual and two modes of tactual letter recognition. Perception \& Psychophysics, 34, 243-249.

ThURLOW, W. R. (1986). Some comparisons of characteristics of alphabetic codes for the deaf-blind. Human Factors, 28, 175-186. 